\title{
A Novel Multivariable MGM $(1, m)$ Direct Prediction Model and Its Optimization
}

\author{
Yuanping Ding and Ye Li (iD \\ College of Information and Management Science, Henan Agricultural University, Zhengzhou 450046, China \\ Correspondence should be addressed to Ye Li; zzliye@163.com
}

Received 30 March 2021; Revised 1 June 2021; Accepted 19 July 2021; Published 29 July 2021

Academic Editor: C. H. Wang

Copyright (c) 2021 Yuanping Ding and Ye Li. This is an open access article distributed under the Creative Commons Attribution License, which permits unrestricted use, distribution, and reproduction in any medium, provided the original work is properly cited.

\begin{abstract}
With regard to the traditional MGM $(1, m)$ model having jumping error in solving process, an MGM $(1, m)$ direct prediction model (denoted as DMGM $(1, m)$ model) is proposed and its solution method is put forward at first. Second, considering the inherent time development trend of system behavior sequence is ignored in the DMGM $(1, m)$ model, the DMGM $(1, m)$ model is optimized by introducing a time polynomial term, and the optimized model can be abbreviated as TPDMGM $(1, m, \varphi)$ model. Subsequently, it is theoretically proved that the TPDMGM $(1, m, \varphi)$ model can achieve mutual transformation with the traditional MGM $(1, m)$ model and the DMGM $(1, m)$ model by adjusting the parameter values. Finally, two case studies about predicting the deformation of foundation pit and Henan's vehicle ownership have been carried out to validate the effectiveness of proposed models. Meanwhile, the MGM $(1, m)$ model and Verhulst model are established for comparison. Results show that the modeling performance of four models from superior to inferior is ranked as TPDMGM $(1, m, \varphi)$ model, DMGM $(1, m)$ model, MGM $(1, m)$ model, and Verhulst model, which on the one hand testifies the correctness of defect analysis of the MGM $(1, m)$ model and on the other hand verifies that the TPDMGM $(1, m, \varphi)$ model has advantages in predicting the system variables with mutual relation, mutual restriction, and time development trend characteristic.
\end{abstract}

\section{Introduction}

Grey system theory is an interdisciplinary scientific area that was first proposed in the 1980s by Professor Deng [1]. Since then, the grey system theory has become quite popular in dealing with system analysis, prediction, decision making, and control with partially known information. The grey prediction model is one of the most important components of grey system theory, which can realize the prediction by excavating the internal rules of accumulated sequence [2]. Nowadays, the grey prediction model has been widely used in various fields such as health care [3], agriculture [4], environment [5], and energy [6].

The GM $(1,1)$ model is the foundation and core of grey prediction models, and it is the simplest and most widely used single variable grey prediction model. Over the past four decades, scholars made a lot of efforts to improve the modeling performance of the GM $(1,1)$ model, which mainly includes three aspects: parameters optimization [7], modeling range expansion [8], and modeling structure improvement [9]. These achievements have improved the prediction accuracy of the GM $(1,1)$ model to a certain extent and enriched the research system of grey prediction theory. By observing the basic forms of the GM $(1,1)$ model and its extended models, it is known that they are only applicable to the system simulation and prediction with one variable. However, the actual economic and social system often contains multiple variables as they restrict and interact with each other [10]. In order to solve this kind of system prediction problem more accurately, Zhai et al. [10] proposed the MGM $(1, m)$ model based on the GM $(1,1)$ model. The multivariable MGM $(1, m)$ model is a natural extension of the GM $(1,1)$ model under $\mathrm{m}$-variables, which is presented in the form of $\mathrm{m}$-variable and first-order ordinary differential equations [10]. Although the MGM $(1, m)$ model has been successfully applied in many cases, there may still be distortions in modeling results. Therefore, scholars have conducted many relevant studies to optimize the MGM 
$(1, m)$ model. In the aspect of modeling rang expansion, due to the continuous improvement of the level of science and technology and the limited cognitive ability of human beings, system information is only known the range instead of the exact value. Hence, Xiong et al. [5] proposed an MGM (1, $m$ ) model for interval grey numbers and applied it to air pollution prediction. In terms of parameter optimization, Dai et al. [11] improved the background value by using a nonhomogeneous exponential function to fit the first-order accumulated sequence. In order to reflect the new information priority principle, $\mathrm{Wu}$ et al. [12] replaced the traditional first-order accumulation generation operator with fractional-order accumulation generation operator in the $\operatorname{MGM}(1, m)$ model and used the particle swarm algorithm to calculate the optimal order. Based on these achievements, the modeling precision of the $\operatorname{MGM}(1, m)$ model in practical application is improved. Through observing the basic forms of these optimized models, it is found that they are mainly suitable for the original data matrix with quasi-exponential law, whereas the modeling performance for the general data sequence is not ideal. For this reason, Wang and Zhao [13] proposed a nonhomogeneous multivariate $\operatorname{MGM}(1, m)$ prediction model, which improved the consistency between modeling data and model structure. In view of the nonlinear relationship between the variables in system, Xiong et al. [14] constructed a nonlinear multivariable $\operatorname{MGM}(1, m)$ prediction model and studied the time lag characteristics of system behaviors.

The works presented above studied the modeling rang expansion, parameter optimization, and modeling structure improvement of the traditional $\operatorname{MGM}(1, m)$ model, and significant findings were obtained that improved the performance of the MGM $(1, m)$ model. However, these optimized MGM $(1, m)$ models still have some defects in two aspects. (1) Parameter defect: there is a jumping error between parameter estimation and parameter application in the MGM (1, m) model. (2) Structure defect: the model structure does not match the characteristics of the system variables. The traditional MGM $(1, m)$ model and its optimization models only consider that there is an interaction and mutual restrict relationship between each variable, while ignoring the inherent time development trend of each variable. For example, in the research of foundation pit deformation prediction, settlement observation points are not only interrelated and interact with each other but also gradually tend to the saturation state as time changes. This practical application background is not reflected in the existing multivariable MGM $(1, m)$ prediction model.

In this paper, the defects of the traditional MGM $(1, m)$ model are systematically studied; based on this, the definition equation of the DMGM $(1, m)$ model is proposed and the recursive formula of the $\operatorname{DMGM}(1, m)$ model is given. On the basis of the DMGM $(1, m)$ model, taking into account the inherent time development trend of system behaviors, an optimized TPDMGM $(1, m, \varphi)$ model is constructed by introducing a time polynomial term. Finally, the validity of proposed models is verified by predicting the deformation of foundation pit and vehicle ownership in Henan province.

The other parts of this research are organized as follows. In Section 2, basic definitions of the MGM $(1, m)$ model and its defects are presented. Section 3 is the detailed modeling procedure of the DMGM $(1, m)$ model. The optimized TPDMGM $(1, m, \varphi)$ model is put forward in Section 4. Section 5 gives two example analyses about predicting the deformation of foundation pit and vehicle ownership in Henan province to illustrate the practicality and effectiveness of proposed models. The last section is devoted to conclusions.

\section{The MGM $(1, m)$ Model and Its Defect Analysis}

The MGM $(1, m)$ model is a grey prediction model with $m$ variables and first-order differential equations [10]. Compared with the traditional grey prediction models with a single variable, such as GM $(1,1)$ model and DGM $(1,1)$ model, the MGM $(1, m)$ model takes into account the interrelationship and mutual restriction between variables on the system. Therefore, it is more useful for system prediction than those for grey prediction models with one variable. The definition of the MGM $(1, m)$ model is as follows.

Definition 1 (see [15]). Let $\mathbf{Y}^{(0)}=\left(\mathbf{y}_{1}^{(0)}, \mathbf{y}_{2}^{(0)}, \ldots, \mathbf{y}_{\mathbf{m}}^{(0)}\right)$ be a nonnegative original data matrix, $\mathbf{y}_{\mathbf{i}}^{(0)}=\left(y_{i}^{(0)}(1)\right.$, $\left.y_{i}^{(0)}(2), \ldots, y_{i}^{(0)}(n)\right), i=1,2, \ldots, m$ be the original data vector, $\mathbf{y}_{\mathbf{i}}^{(1)}=\left(y_{1}^{(1)}(1), y_{2}^{(1)}(2), \ldots, y_{m}^{(1)}(n)\right), i=1,2, \ldots, m$ be the first-order accumulated generation sequence of $y_{\mathbf{i}}^{(0)}$, and $\mathbf{z}_{\mathbf{i}}^{(1)}=\left(z_{i}^{(1)}(2), z_{i}^{(1)}(3), \ldots, z_{i}^{(1)}(n)\right), i=1,2, \ldots, m$ be the mean sequence generated by consecutive neighbors of $\mathbf{y}_{\mathbf{i}}^{(1)}$, then the basic form of the MGM $(1, m)$ model is defined as follows:

$$
\left\{\begin{array}{l}
\frac{\mathrm{d} y_{1}^{(1)}(t)}{\mathrm{d} t}=a_{11} y_{1}^{(1)}(t)+a_{12} y_{2}^{(1)}(t)+\ldots+a_{1 m} y_{m}^{(1)}(t)+b_{1}, \\
\frac{\mathrm{d} y_{2}^{(1)}(t)}{\mathrm{d} t}=a_{21} y_{1}^{(1)}(t)+a_{22} y_{2}^{(1)}(t)+\ldots+a_{2 m} y_{m}^{(1)}(t)+b_{2}, \\
\ldots \\
\frac{\mathrm{d} y_{m}^{(1)}(t)}{\mathrm{d} t}=a_{m 1} y_{1}^{(1)}(t)+a_{m 2} y_{2}^{(1)}(t)+\ldots+a_{m m} y_{m}^{(1)}(t)+b_{m} .
\end{array}\right.
$$

For convenience, equation (1) can be written in the matrix, that is,

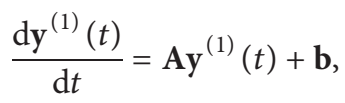

where $\mathbf{A}=\left(a_{i j}\right)_{m \times m}, \mathbf{b}=\left(b_{1}, b_{2}, \ldots, b_{m}\right)^{T}$.

Further, by discretizing equation (1), the discrete form of the MGM $(1, m)$ model is 


$$
\left\{\begin{array}{l}
y_{1}^{(0)}(t)=a_{11} z_{1}^{(1)}(t)+a_{12} z_{2}^{(1)}(t)+\cdots+a_{1 m} z_{m}^{(1)}(t)+b_{1}, \\
y_{2}^{(0)}(t)=a_{21} z_{1}^{(1)}(t)+a_{22} z_{2}^{(1)}(t)+\cdots+a_{2 m} z_{m}^{(1)}(t)+b_{2}, \\
\cdots \\
y_{m}^{(0)}(t)=a_{m 1} z_{1}^{(1)}(t)+a_{m 2} z_{2}^{(1)}(t)+\cdots+a_{m m} z_{m}^{(1)}(t)+b_{m} .
\end{array}\right.
$$

Then, the matrix form of equation (3) can be written as equation (4), which is

$$
\mathbf{y}^{(0)}(t)=\mathbf{A} \mathbf{z}^{(1)}(t)+\mathbf{b}
$$

where $\mathbf{A}=\left(a_{i j}\right)_{m \times m}, \mathbf{b}=\left(b_{1}, b_{2}, \ldots, b_{m}\right)^{T}$.

Theorem 1 (see [10]). Assume that $\mathbf{y}_{\mathbf{i}}^{(0)}, \mathbf{y}_{\mathbf{i}}^{(1)}$, and $\mathbf{z}_{\mathbf{i}}^{(1)}$ are defined as Definition 1 and $\mathbf{p}_{\mathbf{i}}=\left(a_{i 1}, a_{i 2}, \ldots, a_{i m}, b_{i}\right)^{T}$, $i=1,2, \ldots, m$ is parameter vector, then the identification values of parameters vector can be obtained by the least square method, namely,

$$
\widehat{p}_{\mathbf{i}}=\left(\widehat{a}_{i 1}, \widehat{a}_{i 2}, \ldots, \widehat{a}_{i m}, \widehat{b}_{i}\right)^{T}=\left(\mathbf{D}^{T} \mathbf{D}\right)^{-1} \mathbf{D}^{T} \mathbf{l}_{\mathbf{i}}, \quad i=1,2, \ldots, m,
$$

where

$$
\begin{aligned}
\mathbf{D} & =\left[\begin{array}{ccccc}
z_{1}^{(1)}(2) & z_{2}^{(1)}(2) & \ldots & z_{m}^{(1)}(2) & 1 \\
z_{1}^{(1)}(3) & z_{2}^{(1)}(3) & \ldots & z_{m}^{(1)}(3) & 1 \\
\ldots & \ldots & \ldots & \ldots & \ldots \\
z_{1}^{(1)}(n) & z_{2}^{(1)}(n) & \ldots & z_{m}^{(1)}(n) & 1
\end{array}\right], \\
\mathbf{l}_{\mathbf{i}} & =\left[y_{i}^{(0)}(2), y_{i}^{(0)}(3), \ldots, y_{i}^{(0)}(n)\right]^{T}, \quad i=1,2, \ldots, m .
\end{aligned}
$$

Theorem 2. Let the identification values of parameters vector be described in Theorem 1; assume that the initial value $\widehat{\mathbf{y}}^{(1)}(1)=\mathbf{y}^{(1)}(1)=\mathbf{y}^{(0)}(1)$, that is to say, the fitting curve must pass through the first data point of the original data sequence, then the time response function of the multivariable MGM $(1, m)$ model is given as follows:

$$
\widehat{\mathbf{y}}^{(1)}(t)=e^{\mathbf{A}(t-1)}\left(\mathbf{y}^{(1)}(1)+\mathbf{A}^{-1} \mathbf{b}\right)-\mathbf{A}^{-1} \mathbf{b} .
$$

Proof. According to equation (2), we have

$$
\frac{d \mathbf{y}^{(1)}(t)}{\mathbf{A y} \mathbf{y}^{(1)}(t)+\mathbf{b}}=\mathrm{d} t \text {. }
$$

Integrating the whitening differential equation (8), we

$$
\begin{aligned}
\int \frac{1}{\mathbf{A y}{ }^{(1)}(t)+\mathbf{b}} \mathrm{dy}^{(1)}(t) & =\int \mathrm{d} t, \\
\frac{1}{\mathbf{A}} \ln C\left(\mathbf{A} \mathbf{y}^{(1)}(t)+\mathbf{b}\right) & =t, \\
\mathbf{A y}^{(1)}(t)+\mathbf{b} & =C e^{\mathbf{A} t} .
\end{aligned}
$$

Setting $t=1$, we can obtain the value of $C$, that is, $C=e^{-\mathbf{A}}\left(\mathbf{A y}{ }^{(1)}(1)+\mathbf{b}\right)$.

Then, substituting $C$ into equation (9), we obtain the time response function of the MGM $(1, m)$ model as follows:

$$
\widehat{y}^{(1)}(t)=e^{\mathbf{A}(t-1)}\left(\mathbf{y}^{(1)}(1)+\mathbf{A}^{-1} \mathbf{b}\right)-\mathbf{A}^{-1} \mathbf{b} .
$$

The MGM $(1, m)$ model effectively realizes a unified description of multiple variables from the perspective of system and can better describe the mutual influence and mutual restriction among the variables in system. However, the parameters of the MGM $(1, m)$ model are often deduced from equation (3), whereas the time response function is solved by equation (1). The inconsistency between parameters estimation and parameters application is an important factor leading to a poor prediction accuracy of the MGM $(1, m)$ model. Therefore, it is particularly important to construct a multivariable $\operatorname{MGM}(1, m)$ direct prediction model that can unify parameters estimation and parameters application.

\section{Construction and Solution of the DMGM $(1, m)$ Model}

A novel MGM $(1, m)$ direct prediction model (abbreviated as DMGM $(1, m))$ is proposed in this section. The parameter estimation and recursive formula of the DMGM $(1, m)$ model are deduced.

\subsection{Construction of the DMGM $(1, m)$ Model}

Definition 2. Let $\mathbf{X}^{(0)}=\left(\mathbf{x}_{1}^{(0)}, \mathbf{x}_{2}^{(0)}, \ldots, \mathbf{x}_{\mathrm{m}}^{(0)}\right)$ be a nonnegative original data matrix, $\mathbf{x}_{\mathbf{i}}^{(0)}=\left(x_{i}^{(0)}(1), x_{i}^{(0)}(2), \ldots\right.$, $\left.x_{i}^{(0)}(n)\right), i=1,2, \ldots, m$ be the original data vector, $\mathbf{x}_{\mathbf{i}}^{(1)}=$ $\left(x_{i}^{(1)}(1), x_{i}^{(1)}(2), \ldots, x_{i}^{(1)}(n)\right), i=1,2, \ldots, m$ be the firstorder accumulated generation sequence of $\mathbf{x}_{\mathbf{i}}^{(0)}$, and $\mathbf{z}_{\mathbf{i}}^{(1)}=$ $\left(z_{i}^{(1)}(2), z_{i}^{(1)}(3), \ldots, z_{i}^{(1)}(n)\right), i=1,2, \ldots, m$ be the mean sequence generated by consecutive neighbors of $\mathbf{x}_{\mathbf{i}}^{(1)}$, then

$\left\{\begin{array}{l}x_{1}^{(0)}(k)=\beta_{11} x_{1}^{(1)}(k-1)+\beta_{12} z_{2}^{(1)}(k)+\cdots+\beta_{1 m} z_{m}^{(1)}(k)+\omega_{1}, \\ x_{2}^{(0)}(k)=\beta_{21} z_{1}^{(1)}(k)+\beta_{22} x_{2}^{(1)}(k-1)+\ldots+\beta_{2 m} z_{m}^{(1)}(k)+\omega_{2}, \\ \cdots \\ x_{m}^{(0)}(k)=\beta_{m 1} z_{1}^{(1)}(k)+\beta_{m 2} z_{2}^{(1)}(k)+\ldots+\beta_{m m} x_{m}^{(1)}(k-1)+\omega_{m},\end{array}\right.$ get 
is called a multivariable $\mathrm{MGM}(1, m)$ direct prediction model, denoted as DMGM $(1, m)$ model.

\subsection{Solution of the DMGM $(1, m)$ Model}

Theorem 3. Assuming that $\mathbf{x}_{\mathbf{i}}^{(0)}, \mathbf{x}_{\mathbf{i}}^{(1)}$, and $\mathbf{z}_{\mathbf{i}}^{(1)}$ are defined as Definition 2 and $\mathbf{q}_{\mathbf{i}}=\left(\beta_{i 1}, \beta_{i 2}, \ldots, \beta_{i m}, \omega_{i}\right)^{T}, i=1,2, \ldots, m$ is parameters vector, then the identification values of parameters vector can be obtained by the least square method, that is,

$$
\widehat{q}_{\mathbf{i}}=\left(\widehat{\beta}_{i 1}, \widehat{\beta}_{i 2}, \ldots, \widehat{\beta}_{i m}, \widehat{\omega}_{i}\right)^{T}=\left(\mathbf{R}_{\mathbf{i}}^{\mathrm{T}} \mathbf{R}_{\mathbf{i}}\right)^{-1} \mathbf{R}_{\mathbf{i}}^{T} \mathbf{s}_{\mathbf{i}}, \quad i=1,2, \ldots, m .
$$

Taking $i=1$ as an example, then we obtain $\widehat{q}_{1}=\left(\widehat{\beta}_{11}, \widehat{\beta}_{12}, \ldots, \widehat{\beta}_{1 m}, \widehat{\omega}_{1}\right)^{T}=\left(\mathbf{R}_{1}^{\mathrm{T}} \mathbf{R}_{1}\right)^{-1} \mathbf{R}_{1}^{T} \mathbf{s}_{1}$, where

$$
\begin{aligned}
\mathbf{R}_{1} & =\left[\begin{array}{ccccc}
x_{1}^{(1)}(1) & z_{2}^{(1)}(2) & \ldots & z_{m}^{(1)}(2) & 1 \\
x_{1}^{(1)}(2) & z_{2}^{(1)}(3) & \ldots & z_{m}^{(1)}(3) & 1 \\
\ldots & \ldots & \ldots & \ldots & \ldots \\
x_{1}^{(1)}(n-1) & z_{2}^{(1)}(n) & \ldots & z_{m}^{(1)}(n) & 1
\end{array}\right], \\
\mathbf{s}_{1} & =\left[x_{1}^{(0)}(2), x_{1}^{(0)}(3), \ldots, x_{1}^{(0)}(n)\right]^{T} .
\end{aligned}
$$

Similarly, the estimated values of parameters can be solved by the least square method when $i=2,3, \ldots, m$.

Theorem 4. Assume that the identification values of parameter vector of the DMGM $(1, \mathrm{~m})$ model are shown in Theorem 3, the initial value $\widehat{x}_{i}^{(1)}(1)=x_{i}^{(1)}(1)=$ $x_{i}^{(0)}(1), i=1,2, \ldots, m$, that is to say, the fitting curve must pass through the first data point of the original data sequence, then the recursive formula of the DMGM $(1, m)$ model is given as follows:

$$
\left\{\begin{array}{l}
\widehat{x}_{1}^{(0)}(k)=\widehat{\beta}_{11} x_{1}^{(1)}(k-1)+\widehat{\beta}_{12} z_{2}^{(1)}(k)+\cdots+\widehat{\beta}_{1 m} z_{m}^{(1)}(k)+\widehat{\omega}_{1}, \\
\widehat{x}_{2}^{(0)}(k)=\widehat{\beta}_{21} z_{1}^{(1)}(k)+\widehat{\beta}_{22} x_{2}^{(1)}(k-1)+\cdots+\widehat{\beta}_{2 m} z_{m}^{(1)}(k)+\widehat{\omega}_{2}, \\
\cdots \\
\widehat{x}_{m}^{(0)}(k)=\widehat{\beta}_{m 1} z_{1}^{(1)}(k)+\widehat{\beta}_{m 2} z_{2}^{(1)}(k)+\cdots+\widehat{\beta}_{m m} x_{m}^{(1)}(k-1)+\widehat{\omega}_{m} .
\end{array}\right.
$$

3.3. Advantages of the DMGM $(1, m)$ Model. Compared with the traditional MGM $(1, m)$ model, there are three advantages of the DMGM $(1, m)$ model. First, in the aspect of modeling structure, the DMGM $(1, m)$ model is the discrete form of the MGM $(1, m)$ model, which effectively avoids jumping errors and achieves the unification between parameter estimation and parameter application. Second, in the aspect of solving time response function, the DMGM (1, $m$ ) model adopts the recursive iteration method, which avoids the complexity of solving the differential equations of the MGM $(1, m)$ model. More important, the $\operatorname{DMGM}(1, m)$ model can directly give the final fitted value of each variable without inverse accumulated generation operation. In summary, the DMGM $(1, m)$ model not only significantly improves the modeling accuracy in theory but also has the advantage of simple modeling process.

However, it is found that the DMGM $(1, m)$ model can only describe the interrelationship and mutual restriction of system variables from equation (11). Because the DMGM (1, $m$ ) model is the discrete form of the MGM $(1, m)$ model, therefore, the DMGM $(1, m)$ model is suitable for the homogeneous exponential data prediction as the $\operatorname{MGM}(1, m)$ model. In the actual economic and social system, system variables often have a nonhomogeneous exponential characteristic or time power term characteristics, that is to say, system variables have a certain relationship with time term. Hence, considering that the certain relationship between system variables and time items, we will add a time polynomial term into the DMGM $(1, m)$ model. Subsequently, an optimized DMGM $(1, m)$ model, abbreviated as TPDMGM $(1, m, \varphi)$, is proposed in the following section.

\section{Construction and Solution of the TPDMGM $(1, m, \varphi)$ Model}

In this section, we will present the modeling procedure of the TPDMGM $(1, m, \varphi)$ model, including definition equation, property, time response function, and modeling steps.

\subsection{Construction of the TPDMGM $(1, m, \varphi)$ Model}

Definition 3. Let $\mathbf{U}^{(0)}=\left(\mathbf{u}_{1}^{(0)}, \mathbf{u}_{2}^{(0)}, \ldots, \mathbf{u}_{\mathrm{m}}^{(0)}\right)$ be a nonnegative original data matrix, $\mathbf{u}_{\mathbf{i}}^{(0)}=\left(u_{i}^{(0)}(1), u_{i}^{(0)}(2), \ldots\right.$, $\left.u_{i}^{(0)}(n)\right), i=1,2, \ldots, m$ be the original data vector, $\mathbf{u}_{\mathbf{i}}^{(1)}=$ $\left(u_{i}^{(1)}(1), u_{i}^{(1)}(2), \ldots, u_{i}^{(1)}(n)\right), i=1,2, \ldots, m$ be the firstorder accumulated generation sequence of $\mathbf{u}_{\mathbf{i}}^{(0)}$, and $\mathbf{z}_{\mathbf{i}}^{(1)}=$ $\left(z_{i}^{(1)}(2), z_{i}^{(1)}(3), \ldots, z_{i}^{(1)}(n)\right), i=1,2, \ldots, m$ be the mean sequence generated by consecutive neighbors of $\mathbf{u}_{\mathbf{i}}^{(1)}$, then

$$
\left\{\begin{array}{l}
u_{1}^{(0)}(k)=\gamma_{11} u_{1}^{(1)}(k-1)+\gamma_{12} z_{2}^{(1)}(k)+\cdots+\gamma_{1 m} z_{m}^{(1)}(k)+\theta_{10}+\theta_{11} t+\theta_{12} t^{2}+\ldots+\theta_{1 \varphi} t^{\varphi}, \\
u_{2}^{(0)}(k)=\gamma_{21} z_{1}^{(1)}(k)+\gamma_{22} u_{2}^{(1)}(k-1)+\cdots+\gamma_{2 m} z_{m}^{(1)}(k)+\theta_{20}+\theta_{21} t+\theta_{22} t^{2}+\cdots+\theta_{2 \varphi} t^{\varphi}, \\
\cdots \\
u_{m}^{(0)}(k)=\gamma_{m 1} z_{1}^{(1)}(k)+\gamma_{m 2} z_{2}^{(1)}(k)+\cdots+\gamma_{m m} u_{m}^{(1)}(k-1)+\theta_{m 0}+\theta_{m 1} t+\theta_{m 2} t^{2}+\cdots+\theta_{m \varphi} t^{\varphi},
\end{array}\right.
$$


is called a DMGM $(1, m)$ model with time polynomial term, abbreviated as TPDMGM $(1, m, \varphi)$ model.

In this model, $\theta_{11} t+\theta_{12} t^{2}+\cdots+\theta_{1 \varphi} t^{\varphi}$ is the time polynomial term that reflects the relationship between system behaviors with time items. Additionally, it is worth noting that when $\varphi=0$, the TPDMGM $(1, m, \varphi)$ model is transformed into the DMGM $(1, m)$ model, and when $\varphi=0, \gamma_{i i}=\left(a_{i i} / 1-0.5 a_{i i}\right), i=1,2, \ldots, 3$, the TPDMGM ( 1 , $m, \varphi$ ) model is degraded into the MGM $(1, m)$ model (see Property 1 and Property 2), indicating that the TPDMGM (1, $m, \varphi)$ model has wider applicable fields than the traditional MGM $(1, m)$ model. The transformation relationships among these models are proved as follows.

Property 1 . When $\varphi=0$, the TPDMGM $(1, m, \varphi)$ model is equal to the DMGM $(1, m)$ model.

Proof. When $\varphi=0$, according to Definition 3, we can obtain

$$
\left\{\begin{array}{l}
u_{1}^{(0)}(k)=\gamma_{11} u_{1}^{(1)}(k-1)+\gamma_{12} z_{2}^{(1)}(k)+\cdots+\gamma_{1 m} z_{m}^{(1)}(k)+\theta_{10}, \\
u_{2}^{(0)}(k)=\gamma_{21} z_{1}^{(1)}(k)+\gamma_{22} u_{2}^{(1)}(k-1)+\cdots+\gamma_{2 m} z_{m}^{(1)}(k)+\theta_{20}, \\
\cdots \\
u_{m}^{(0)}(k)=\gamma_{m 1} z_{1}^{(1)}(k)+\gamma_{m 2} z_{2}^{(1)}(k)+\cdots+\gamma_{m m} u_{m}^{(1)}(k-1)+\theta_{m 0} .
\end{array}\right.
$$

Obviously, equation (16) is equal to equation (11), that is, the DMGM $(1, m)$ model. Proof ends.

Property 2. When $\varphi=0, \gamma_{i i}=\left(a_{i i} / 1-0.5 a_{i i}\right), i=1,2, \ldots, 3$, the TPDMGM $(1, m, \varphi)$ model is the traditional multivariable MGM $(1, m)$ model.

Proof. When $\varphi=0, \gamma_{i i}=\left(a_{i i} / 1-0.5 a_{i i}\right), i=1,2, \ldots, 3$, according to Definition 3, we have

$$
\left\{\begin{array}{l}
\left(1-0.5 a_{11}\right) u_{1}^{(0)}(k)=a_{11} u_{1}^{(1)}(k-1)+\left(1-0.5 a_{11}\right) \gamma_{12} z_{2}^{(1)}(k)+\cdots \\
+\left(1-0.5 a_{11}\right) \gamma_{1 m} z_{m}^{(1)}(k)+\left(1-0.5 a_{11}\right) \theta_{10}, \\
\left(1-0.5 a_{22}\right) u_{2}^{(0)}(k)=\left(1-0.5 a_{22}\right) \gamma_{21} z_{1}^{(1)}(k)+a_{22} u_{2}^{(1)}(k-1)+\ldots \\
+\left(1-0.5 a_{22}\right) \gamma_{2 m} z_{m}^{(1)}(k)+\left(1-0.5 a_{22}\right) \theta_{20}, \\
\cdots \\
\left(1-0.5 a_{m m}\right) u_{m}^{(0)}(k)=\left(1-0.5 a_{m m}\right) \gamma_{m 1} z_{1}^{(1)}(k)+\left(1-0.5 a_{m m}\right) \gamma_{m 2} z_{2}^{(1)}(k)+\cdots \\
+a_{m m} u_{m}^{(1)}(k-1)+\left(1-0.5 a_{m m}\right) \theta_{m 0} .
\end{array}\right.
$$

Since $z_{i}^{(1)}(k)=\left(u_{i}^{(1)}(k)+u_{i}^{(1)}(k-1) / 2\right)=\left(2 u_{i}^{(1)}(k-1)\right.$ $\left.+u_{i}^{(0)}(k) / 2\right)=u_{i}^{(1)}(k-1)+0.5 u_{i}^{(0)}(k)$, then we obtain

$$
\left\{\begin{array}{l}
u_{1}^{(0)}(k)=a_{11} z_{1}^{(1)}(k)+\left(1-0.5 a_{11}\right) \gamma_{12} z_{2}^{(1)}(k)+\cdots+\left(1-0.5 a_{11}\right) \gamma_{1 m} z_{m}^{(1)}(k)+\left(1-0.5 a_{11}\right) \theta_{10}, \\
u_{2}^{(0)}(k)=\left(1-0.5 a_{22}\right) \gamma_{21} z_{1}^{(1)}(k)+a_{22} z_{2}^{(1)}(k)+\cdots+\left(1-0.5 a_{22}\right) \gamma_{2 m} z_{m}^{(1)}(k)+\left(1-0.5 a_{22}\right) \theta_{20}, \\
\cdots \\
u_{m}^{(0)}(k)=\left(1-0.5 a_{m m}\right) \gamma_{m 1} z_{1}^{(1)}(k)+\left(1-0.5 a_{m m}\right) \gamma_{m 2} z_{2}^{(1)}(k)+\cdots+a_{m m} z_{m}^{(1)}(k)+\left(1-0.5 a_{m m}\right) \theta_{m 0} .
\end{array}\right.
$$

Finally, equation (18) can be further simplified as

$$
\left\{\begin{array}{l}
u_{1}^{(0)}(k)=a_{11} z_{1}^{(1)}(k)+a_{12} z_{2}^{(1)}(k)+\cdots+a_{1 m} z_{m}^{(1)}(k)+b_{1} \\
u_{2}^{(0)}(k)=a_{21} z_{1}^{(1)}(k)+a_{22} z_{2}^{(1)}(k)+\cdots+a_{2 m} z_{m}^{(1)}(k)+b_{2} \\
\cdots \\
u_{m}^{(0)}(k)=a_{m 1} z_{1}^{(1)}(k)+a_{m 2} z_{2}^{(1)}(k)+\cdots+a_{m m} z_{m}^{(1)}(k)+b_{m}
\end{array}\right.
$$

which is the traditional MGM $(1, m)$ model. Proof ends.

\subsection{Solution of the TPDMGM $(1, m, \varphi)$ Model}

Theorem 5. Let $\mathbf{u}_{\mathbf{i}}^{(0)}, \mathbf{u}_{\mathbf{i}}^{(1)}$, and $\mathbf{z}_{\mathbf{i}}^{(1)}$ be defined as Definition 3 , then the identification values of parameters vector $\mathbf{w}_{\mathbf{i}}=\left(\gamma_{i 1}, \gamma_{i 2}, \ldots, \gamma_{i m}, \theta_{i 0}, \theta_{i 1}, \ldots, \theta_{i \varphi}\right)^{T}, i=1,2, \ldots, m$ can be obtained by the least square method, that is,

$$
\begin{aligned}
\widehat{w}_{\mathbf{i}} & =\left(\widehat{\gamma}_{i 1}, \widehat{\gamma}_{i 2}, \ldots, \widehat{\gamma}_{i m}, \widehat{\theta}_{i 0}, \widehat{\theta}_{i 1}, \ldots, \widehat{\theta}_{i \varphi}\right)^{T} \\
& =\left(\mathbf{G}_{\mathbf{i}}^{T} \mathbf{G}_{\mathbf{i}}\right)^{-1} \mathbf{G}_{\mathbf{i}}^{T} \mathbf{f}_{i}, \quad i=1,2, \ldots, m .
\end{aligned}
$$


Taking $i=1$ as an example, then we obtain $\widehat{w}_{1}=\left(\gamma_{11}, \gamma_{12}, \ldots, \gamma_{1 m}, \theta_{10}, \theta_{11}, \ldots, \theta_{1 \varphi}\right)^{T}=\left(\mathbf{G}_{1}^{T} \mathbf{G}_{1}\right)^{-1} \mathbf{G}_{1}^{T} \mathbf{f}_{1}$, where

$$
\begin{aligned}
& \mathbf{G}_{1}=\left[\begin{array}{ccccccccc}
u_{1}^{(1)}(1) & z_{2}^{(1)}(2) & \ldots & z_{m}^{(1)}(2) & 1 & 2 & 2^{2} & \ldots & 2^{\varphi} \\
u_{1}^{(1)}(2) & z_{2}^{(1)}(3) & \ldots & z_{m}^{(1)}(3) & 1 & 3 & 3^{2} & \ldots & 3^{\varphi} \\
\ldots & \ldots & \ldots & \ldots & \ldots & \ldots & \ldots & \ldots & \ldots \\
u_{1}^{(1)}(n-1) & z_{2}^{(1)}(n) & \ldots & z_{m}^{(1)}(n) & 1 & n & n^{2} & \ldots & n^{\varphi}
\end{array}\right], \\
& \mathbf{f}_{1}=\left[u_{1}^{(0)}(2), u_{1}^{(0)}(3), \ldots, u_{1}^{(0)}(n)\right]^{T} \text {. }
\end{aligned}
$$

Similarly, the estimated values of parameters can be solved by the least square method when $i=2,3, \ldots, m$.

However, as can be seen in equation (21), if we want to solve the estimated values of parameters, we should calculate the value of $\varphi$ at first. Taking into account the volatility characteristics of high-order polynomials and the principle of thrift (the more complex the model, the higher the probability of overfitting) [16], the value of parameter $\varphi$ is limited to $\{0,1,2,3\}$. In practical applications, the minimum average relative error is used as the criterion and the debugging method is used to solve the optimal value of $\varphi$.

Theorem 6. Assume that the identification values of parameters of the TPDMGM $(1, m, \varphi)$ model are shown in Theorem 5 and the initial value $\widehat{u}_{i}^{(1)}(1)=u_{i}^{(1)}(1)=u_{i}^{(0)}(1), i=1,2, \ldots, m$, then the recursive formula of the TPDMGM $(1, m, \varphi)$ model is given as follows:

$$
\left\{\begin{array}{l}
\widehat{u}_{1}^{(0)}(k)=\widehat{\gamma}_{11} u_{1}^{(1)}(k-1)+\widehat{\gamma}_{12} z_{2}^{(1)}(k)+\cdots+\widehat{\gamma}_{1 m} z_{m}^{(1)}(k)+\widehat{\theta}_{10}+\widehat{\theta}_{11} t+\widehat{\theta}_{12} t^{2}+\cdots+\widehat{\theta}_{1 \varphi} t^{\varphi}, \\
\widehat{u}_{2}^{(0)}(k)=\widehat{\gamma}_{21} z_{1}^{(1)}(k)+\widehat{\gamma}_{22} u_{2}^{(1)}(k-1)+\cdots+\widehat{\gamma}_{2 m} z_{m}^{(1)}(k)+\widehat{\theta}_{20}+\widehat{\theta}_{21} t+\widehat{\theta}_{22} t^{2}+\cdots+\widehat{\theta}_{2 \varphi} t^{\varphi}, \\
\cdots \\
\widehat{u}_{m}^{(0)}(k)=\widehat{\gamma}_{m 1} z_{1}^{(1)}(k)+\widehat{\gamma}_{m 2} z_{2}^{(1)}(k)+\cdots+\widehat{\gamma}_{m m} u_{m}^{(1)}(k-1)+\widehat{\theta}_{m 0}+\widehat{\theta}_{m 1} t+\widehat{\theta}_{m 2} t^{2}+\cdots+\widehat{\theta}_{m \varphi} t^{\varphi} .
\end{array}\right.
$$

4.3. Advantages of the TPDMGM $(1, m, \varphi)$ Model. From the detailed modeling procedure and property analysis above, we can see that the TPDMGM $(1, m, \varphi)$ model has two modeling advantages. First, the TPDMGM $(1, m, \varphi)$ model can not only realize the unification of parameter estimation and parameter application but also describe the relationship between system behaviors and time term by adjusting the time polynomial term value, which effectively makes up for the theoretical defect and structure defect of the traditional MGM $(1, m)$ model and the DMGM $(1, m)$ model. Next, it is theoretically proved that the DMGM $(1, m)$ model and the MGM $(1, m)$ model are the special forms of the TPDMGM $(1, m, \varphi)$ model, indicating that the TPDMGM $(1, m, \varphi)$ model is compatible.

4.4. Modeling Steps of the TPDMGM (1, $m, \varphi)$ Model. According to the above studies of the TPDMGM $(1, m, \varphi)$ model, the whole modeling and optimization process are briefly described as follows:

Step 1. System variables selection: system variables that influence, correlate, and restrict with each other are selected.

Step 2. Model construction: the TPDMGM (1, $m, \varphi)$ model is established according to equation (15) when the system variables have time development trend characteristic; otherwise, the DMGM $(1, m)$ model is built through equation (11).

Step 3. Parameter estimation: the debugging method is used to solve the value of $\varphi$, and the least square method is used to estimate the value of parameters.
Step 4. Simulation and prediction: the parameter values in step 3 are substituted into the recursive formula and then the simulation and prediction values of system variables are computed.

Step 5. Accuracy test: the mean relative simulation and prediction percentage errors are calculated.

Note. There are two methods to testify whether the system variables have a time development trend characteristic. (1) Quantitative analysis: Before modeling, MATLAB can be used to fit the original data sequence, and then the fitting functions of data sequences are obtained. According to the function, if it is a homogeneous exponential function, the $\operatorname{DMGM}(1, m)$ model will be used for prediction; otherwise, the TPDMGM $(1, m, \varphi)$ model will be used for prediction. (2) Qualitative analysis: In the actual economic and social system, system variables cannot simply be expressed by the homogeneous exponential growth law. These system variables often have a nonhomogeneous exponential characteristic or time power term characteristics, that is to say, system variables have a certain relationship with time term. Therefore, most system variables have a time development trend and can be predicted by the TPDMGM $(1, m, \varphi)$ model.

To clearly illustrate the procedure of TPDMGM $(1, m, \varphi)$ model construction, a flowchart is drawn in Figure 1.

\section{Case Study and Analysis}

In this section, two case studies about forecasting the deformation of foundation pit and Henan's vehicle ownership are given as follows. 


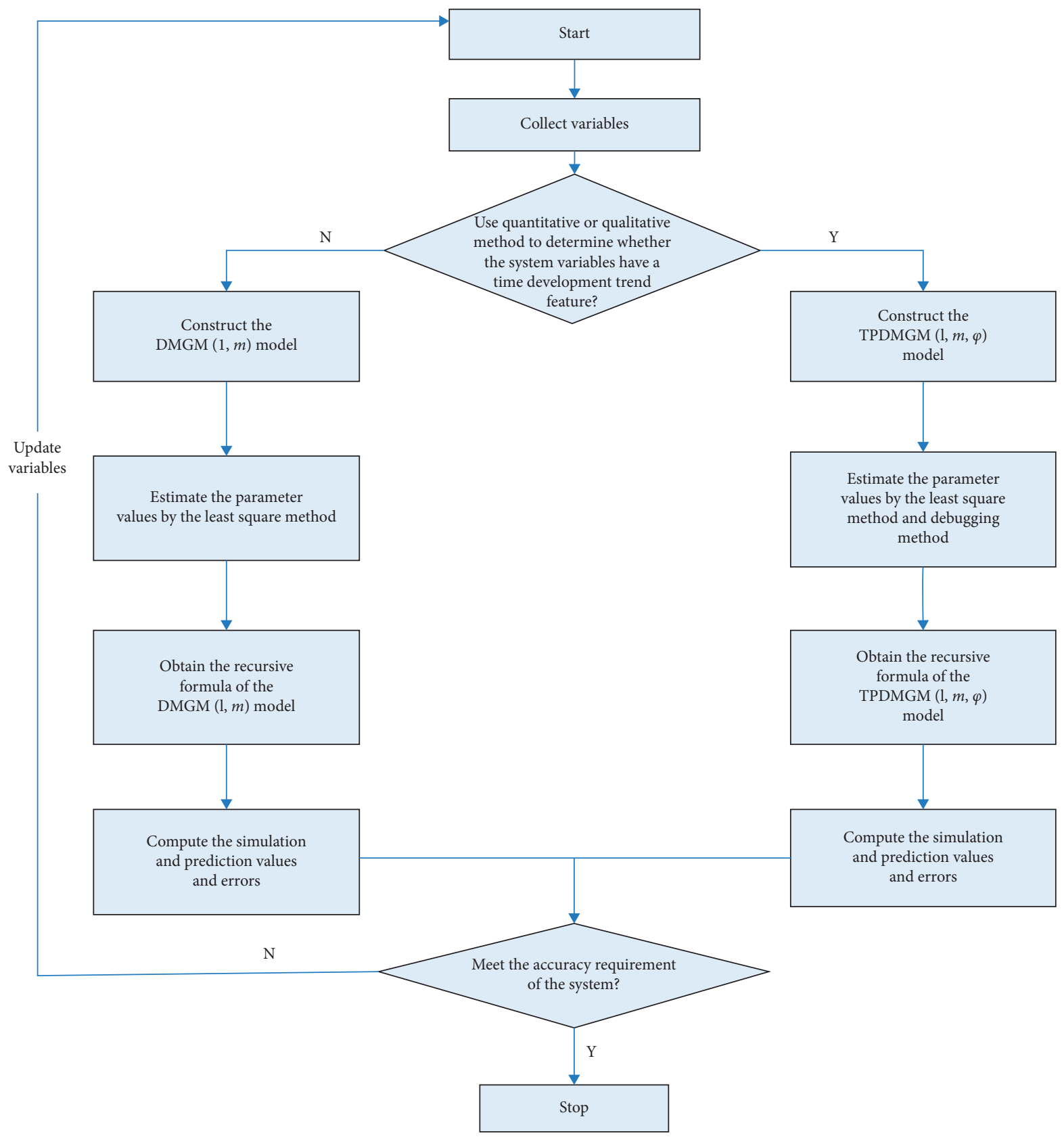

FIgURE 1: The flowchart of the TPDMGM $(1, m, \varphi)$ model construction.

5.1. Case 1: Forecasting the Deformation of Foundation Pit. In order to verify the efficiency and practicality of the proposed DMGM $(1, m)$ model and TPDMGM $(1, m, \varphi)$ model, three groups of original data sequences that are representative and truly reflect the deformation law of foundation pits in Feng et al. [17] are selected in this paper for modeling. The original data are shown in Table 1 .

In our modeling process, we use the first seven data for modeling and the last two data for testing. To illustrate the performance of the DMGM $(1, m)$ model and the TPDMGM $(1, m, \varphi)$ model, the simulation and prediction errors of these models are computed and compared with the commonly used MGM $(1, m)$ model and Verhulst model. The meanings of symbols $\hat{x}_{i}^{(0)}(k), \Delta_{s}(k), \Delta_{p}(k), \bar{\Delta}_{s}, \bar{\Delta}_{p}, \bar{\Delta}_{A s}$, and $\bar{\Delta}_{A p}$ are given in Table 2 . The detailed modeling results are shown in Table $3-5$, as follows.

From these results in Tables $3-5$, we can see that the TPDMGM $(1,3,1)$ model, both in prediction and simulation, has the smallest errors among four models, the performance of the DMGM $(1,3)$ model is slightly inferior to the TPDMGM $(1,3,1)$ model but better than that of the MGM $(1,3)$ model. Comparatively, the results generated by the Verhulst model are the worst among four models. The overall average relative simulation and prediction percentage errors of the TPDMGM $(1,3,1)$ model are $0.0594 \%$ and $0.9029 \%$, while the DMGM $(1,3), \operatorname{MGM}(1,3)$, and Verhulst models are $0.0644 \%$ and $0.9428 \%, 0.8108 \%$ and $1.8119 \%$, and $3.4344 \%$ and $3.9838 \%$, respectively. In summary, the 
TABle 1: Three groups of original data series of foundation pit deformation.

\begin{tabular}{lccr}
\hline$k$ & $x_{1}$ & $x_{2}$ & $x_{3}$ \\
\hline 1 & 8.48 & 9.29 & 10.07 \\
2 & 12.77 & 13.67 & 14.52 \\
3 & 15.10 & 16.23 & 17.28 \\
4 & 17.87 & 19.00 & 20.05 \\
5 & 19.66 & 20.84 & 21.84 \\
6 & 22.30 & 23.33 & 24.28 \\
7 & 24.32 & 25.39 & 26.34 \\
8 & 26.10 & 27.22 & 28.15 \\
9 & 28.90 & 29.35 & 30.40 \\
\hline
\end{tabular}

TABLE 2: The meanings of mathematical symbols.

\begin{tabular}{lc}
\hline Symbols & Meanings \\
\hline$\hat{x}_{i}^{(0)}(k)$ & Simulation or prediction values \\
$\Delta_{s}(k)$ & Relative simulation percentage error of $\hat{x}_{i}^{(0)}(k)$ \\
$\Delta_{p}(k)$ & Relative prediction percentage error of $\hat{x}_{i}^{(0)}(k)$ \\
$\bar{\Delta}_{s}$ & Average relative simulation percentage error for each variable \\
$\bar{\Delta}_{p}$ & Average relative prediction percentage error for each variable \\
$\bar{\Delta}_{A s}$ & Overall average relative simulation percentage error \\
$\bar{\Delta}_{A p}$ & Overall average relative prediction percentage error \\
\hline
\end{tabular}

TABle 3: Comparisons of the simulation results of four models.

\begin{tabular}{|c|c|c|c|c|c|c|c|}
\hline$k$ & & 2 & 3 & 4 & 5 & 6 & 7 \\
\hline \multirow{6}{*}{ MGM $(1,3)$} & \multirow{4}{*}{$\widehat{x}_{i}^{(0)}(k)$} & 12.8217 & 15.1825 & 17.5811 & 19.8958 & 22.0854 & 24.2201 \\
\hline & & 13.7660 & 16.2634 & 18.7244 & 21.0289 & 23.1606 & 25.2362 \\
\hline & & 14.6617 & 17.2628 & 19.759 & 22.0381 & 24.1131 & 26.1461 \\
\hline & & $0.4049 \%$ & $0.5464 \%$ & $1.6167 \%$ & $1.1994 \%$ & $0.9623 \%$ & $0.4108 \%$ \\
\hline & \multirow[t]{2}{*}{$\Delta_{s}(k)$} & $0.7023 \%$ & $0.2058 \%$ & $1.4505 \%$ & $0.9064 \%$ & $0.7261 \%$ & $0.6058 \%$ \\
\hline & & $0.9759 \%$ & $0.0995 \%$ & $1.4514 \%$ & $0.9071 \%$ & $0.6874 \%$ & $0.7361 \%$ \\
\hline \multirow{6}{*}{ DMGM $(1,3)$} & \multirow{4}{*}{$\hat{x}_{i}^{(0)}(k)$} & 12.7855 & 15.0894 & 17.8446 & 19.6593 & 22.3439 & 24.2972 \\
\hline & & 13.6673 & 16.2307 & 19.0034 & 20.8486 & 23.3129 & 25.3971 \\
\hline & & 14.5227 & 17.2842 & 20.0410 & 21.8225 & 24.3121 & 26.3274 \\
\hline & & $0.1214 \%$ & $0.0700 \%$ & $0.1421 \%$ & $0.0034 \%$ & $0.1970 \%$ & $0.0938 \%$ \\
\hline & \multirow[t]{2}{*}{$\Delta_{s}(k)$} & $0.0197 \%$ & $0.0044 \%$ & $0.0176 \%$ & $0.0413 \%$ & $0.0732 \%$ & $0.0279 \%$ \\
\hline & & $0.0189 \%$ & $0.0242 \%$ & $0.0447 \%$ & $0.0800 \%$ & $0.1324 \%$ & $0.0480 \%$ \\
\hline \multirow{6}{*}{ TPDMGM $(1,3,1)$} & \multirow{3}{*}{$\widehat{x}_{i}^{(0)}(k)$} & 12.7703 & 15.1140 & 17.8427 & 19.6547 & 22.3349 & 24.3033 \\
\hline & & 13.6708 & 16.2243 & 19.0061 & 20.8473 & 23.3157 & 25.3958 \\
\hline & & 14.5162 & 17.2959 & 20.0349 & 21.8285 & 24.3035 & 26.3309 \\
\hline & \multirow{3}{*}{$\Delta_{s}(k)$} & $0.0023 \%$ & $0.0928 \%$ & $0.1527 \%$ & $0.0270 \%$ & $0.1567 \%$ & $0.0685 \%$ \\
\hline & & $0.0060 \%$ & $0.0352 \%$ & $0.0322 \%$ & $0.0352 \%$ & $0.0614 \%$ & $0.0227 \%$ \\
\hline & & $0.0262 \%$ & $0.0922 \%$ & $0.0752 \%$ & $0.0525 \%$ & $0.0967 \%$ & $0.0344 \%$ \\
\hline \multirow{6}{*}{ Verhulst } & \multirow{3}{*}{$\hat{x}_{i}^{(0)}(k)$} & 11.3120 & 14.3800 & 17.3938 & 20.0826 & 22.2832 & 23.9605 \\
\hline & & 12.3139 & 15.5239 & 18.6048 & 21.2887 & 23.4365 & 25.0413 \\
\hline & & 13.2580 & 16.5786 & 19.7004 & 22.3649 & 24.4576 & 25.9967 \\
\hline & \multirow{3}{*}{$\Delta_{s}(k)$} & $11.4174 \%$ & $4.7682 \%$ & $2.6648 \%$ & $2.1495 \%$ & $0.0753 \%$ & $1.4782 \%$ \\
\hline & & $9.9203 \%$ & $4.3506 \%$ & $2.0800 \%$ & $2.1531 \%$ & $0.4565 \%$ & $1.3734 \%$ \\
\hline & & $8.6915 \%$ & $4.0590 \%$ & $1.7436 \%$ & $2.4034 \%$ & $0.7315 \%$ & $1.3033 \%$ \\
\hline
\end{tabular}

TPDMGM $(1,3,1)$ model has the best modeling performance among these four models.

5.2. Case 2: Forecasting the Vehicle Ownership in Henan Province. In the system for predicting vehicle ownership, vehicle ownership $\left(x_{1}\right)$, gross domestic product (shorted as GDP) $\left(x_{2}\right)$, and population $\left(x_{3}\right)$ are three variables that influence and restrict each other [11]. Therefore, we use the vehicle ownership prediction in Henan province to testify the performance of the DMGM $(1, m)$ model and the TPDMGM $(1, m, \varphi)$ model. The raw data of $x_{1} \sim x_{3}$ from 2012 2019, presented in Table 6, are obtained from Henan statistical yearbook [18]. Next, we use the first seven data for modeling and the last data for testing. 
TABLE 4: Comparisons of the prediction results of four models.

\begin{tabular}{|c|c|c|c|}
\hline & & 8 & 9 \\
\hline \multirow{6}{*}{ MGM $(1,3)$} & \multirow{4}{*}{$\widehat{x}_{i}^{(0)}(k)$} & 26.4887 & 29.1738 \\
\hline & & 27.5014 & 30.2940 \\
\hline & & 28.4352 & 31.3641 \\
\hline & & $1.4893 \%$ & $0.9474 \%$ \\
\hline & \multirow{2}{*}{$\Delta_{p}(k)$} & $1.0338 \%$ & $3.2163 \%$ \\
\hline & & $1.0131 \%$ & $3.1714 \%$ \\
\hline \multirow{6}{*}{ DMGM $(1,3)$} & \multirow{3}{*}{$\widehat{x}_{i}^{(0)}(k)$} & 26.0952 & 28.1797 \\
\hline & & 27.2179 & 29.6558 \\
\hline & & 28.1668 & 29.7808 \\
\hline & \multirow{3}{*}{$\Delta_{p}(k)$} & $0.0186 \%$ & $2.4924 \%$ \\
\hline & & $0.0079 \%$ & $1.0418 \%$ \\
\hline & & $0.0597 \%$ & $2.0367 \%$ \\
\hline \multirow{6}{*}{ TPDMGM $(1,3,1)$} & \multirow{4}{*}{$\widehat{x}_{i}^{(0)}(k)$} & 26.1178 & 28.1823 \\
\hline & & 27.2123 & 29.6450 \\
\hline & & 28.1733 & 29.8682 \\
\hline & & $0.0683 \%$ & $2.4834 \%$ \\
\hline & \multirow{2}{*}{$\Delta_{p}(k)$} & $0.0284 \%$ & $1.0052 \%$ \\
\hline & & $0.0827 \%$ & $1.7494 \%$ \\
\hline \multirow{6}{*}{ Verhulst } & \multirow{4}{*}{$\widehat{x}_{i}^{(0)}(k)$} & 26.0102 & 26.5764 \\
\hline & & 26.9589 & 27.4782 \\
\hline & & 27.8041 & 28.2862 \\
\hline & & $0.3441 \%$ & $8.0401 \%$ \\
\hline & \multirow{2}{*}{$\Delta_{p}(k)$} & $0.9592 \%$ & $6.3775 \%$ \\
\hline & & $1.2288 \%$ & $6.9533 \%$ \\
\hline
\end{tabular}

TABLE 5: Comparisons of the average simulation/prediction errors of four models.

\begin{tabular}{llllll}
\hline Model & $x_{i}$ & $\bar{\Delta}_{s}(\%)$ & $\bar{\Delta}_{A s}(\%)$ & $\bar{\Delta}_{p}(\%)$ & $\bar{\Delta}_{A p}(\%)$ \\
\hline \multirow{2}{*}{ MGM $(1,3)$} & $x_{1}$ & 0.8567 & & 1.2183 & \\
& $x_{2}$ & 0.7661 & 0.8108 & 2.1251 & 1.8119 \\
& $x_{3}$ & 0.8096 & & 2.0923 & \\
\hline \multirow{2}{*}{ DMGM (1, 3) } & $x_{1}$ & 0.1046 & & 1.2555 & \\
& $x_{2}$ & 0.0307 & 0.0644 & 0.5249 & 0.9428 \\
& $x_{3}$ & 0.0580 & & 1.0482 & \\
\multirow{2}{*}{ TPDMGM $(1,3,1)$} & $x_{1}$ & 0.0833 & & 1.2758 & \\
& $x_{2}$ & 0.0321 & $\mathbf{0 . 0 5 9 4}$ & 0.5168 & $\mathbf{0 . 9 0 2 9}$ \\
& $x_{3}$ & 0.0629 & & 0.9160 & \\
Verhulst & $x_{1}$ & 3.7589 & & 4.1921 & \\
& $x_{2}$ & 3.3890 & 3.4344 & 3.6684 & 3.9838 \\
& $x_{3}$ & 3.1554 & & 4.0910 & \\
\hline
\end{tabular}

Meanwhile, we establish the classical MGM $(1, m)$ model and single variable Verhulst grey prediction model to predict vehicle ownership in Henan province for comparison. Their detailed simulation and prediction results are shown in Tables 7-9, as follows.

From Tables 7-9, we know that the average relative errors in simulation and prediction of the TPDMGM $(1,3,1)$ model are $0.0801 \%$ and $0.7143 \%$; the modeling accuracy of the DMGM $(1,3)$ model is slightly lower than that of the TPDMGM $(1,3,1)$ model, which is $0.3983 \%$ and $1.4449 \%$. However, the average relative errors in simulation and prediction of the MGM $(1,3)$ model and Verhulst model are $0.8768 \%, 3.4811 \%$, and $0.6080 \%, 9.8347 \%$, which are worse than the modeling accuracy of the TPDMGM $(1,3,1)$ model and DMGM $(1,3)$ model. The results show that both the simulation and prediction performance of the TPDMGM (1, $3,1)$ model are the best among these four models.

5.3. Analysis of Comparison Results. The errors of four models in two case studies are shown in Table 10, and the comparison results are analyzed as follows.

As shown in Table 10, the modeling performance of four models in two cases from superior to inferior is ranked as the TPDMGM $(1, m, \varphi)$ model, DMGM $(1, m)$ model, MGM $(1, m)$ model, and Verhulst model. The Verhulst model is a single variable grey prediction model, while the other three models are multivariable grey prediction models. Although the Verhulst model is a commonly used grey prediction model for forecasting the sequence with saturated state, it has a low precision in this study because it cannot describe the variables with mutual correlation and mutual restriction characteristic. The MGM $(1, m)$ model can make up for the shortcoming of the Verhulst model; however, the modeling precision is relatively low due to the jumping error between parameter solution and parameter application. The novel proposed DMGM $(1, m)$ model avoids the jumping error in the MGM $(1, m)$ model by unifying the parameter estimation and application into a discrete form, which improves the modeling accuracy. However, the DMGM $(1, m)$ model is only as suitable for predicting homogeneous exponential sequence as the traditional MGM (1, $m$ ) model. Considering the system variables in the actual economic and social system often have a nonhomogeneous exponential characteristic or time power term characteristics, that is to say, system variables have a certain relationship with time term, this paper introduces a time polynomial term into the DMGM $(1, m)$ model and constructs the TPDMGM 
TABle 6: Raw data of the vehicle ownership system.

\begin{tabular}{cccc}
\hline Year & $x_{1}$ & $x_{2}$ & $x_{3}$ \\
\hline 2012 & 5296737 & 28961.92 & 10543 \\
2013 & 6282190 & 31632.5 & 10601 \\
2014 & 7743731 & 34574.76 & 10662 \\
2015 & 8667600 & 37084.1 & 10722 \\
2016 & 10100102 & 40249.34 & 10788 \\
2017 & 11668151 & 44824.92 & 10853 \\
2018 & 13273585 & 49935.9 & 10906 \\
2019 & 14800767 & 54259.2 & 10952 \\
\hline
\end{tabular}

TABLE 7: Comparisons of the simulation results of four models.

\begin{tabular}{|c|c|c|c|c|c|c|c|}
\hline$k$ & & 2 & 3 & 4 & 5 & 6 & 7 \\
\hline \multirow{6}{*}{ MGM $(1,3)$} & \multirow{4}{*}{$\hat{x}_{i}^{(0)}(k)$} & 6348760 & 7543400 & 8774100 & 10072300 & 11486200 & 13089200 \\
\hline & & 31711 & 34184 & 36924.1 & 40090 & 43922 & 48801 \\
\hline & & 10594 & 10647 & 10704 & 10764 & 10822 & 10872 \\
\hline & & $1.0597 \%$ & $2.5870 \%$ & $1.2287 \%$ & $0.2753 \%$ & $1.5594 \%$ & $1.3891 \%$ \\
\hline & \multirow{2}{*}{$\Delta_{s}(k)$} & $0.2482 \%$ & $1.1302 \%$ & $0.4315 \%$ & $0.3959 \%$ & $2.0143 \%$ & $2.2727 \%$ \\
\hline & & $0.0651 \%$ & $0.1444 \%$ & $0.1669 \%$ & $0.2215 \%$ & $0.2838 \%$ & $0.3081 \%$ \\
\hline \multirow{6}{*}{ DMGM $(1,3)$} & \multirow{3}{*}{$\widehat{x}_{i}^{(0)}(k)$} & 6289331 & 7659512 & 8797799 & 10112187 & 11554349 & 13322181 \\
\hline & & 31872 & 34160 & 37042 & 40542 & 44829 & 49857 \\
\hline & & 10602 & 10660 & 10723 & 10788 & 10852 & 10906 \\
\hline & \multirow{3}{*}{$\Delta_{s}(k)$} & $0.1137 \%$ & $1.0876 \%$ & $1.5021 \%$ & $0.1197 \%$ & $0.9753 \%$ & $0.3661 \%$ \\
\hline & & $0.7562 \%$ & $1.1996 \%$ & $0.1131 \%$ & $0.7261 \%$ & $0.0102 \%$ & $0.1589 \%$ \\
\hline & & $0.0055 \%$ & $0.0152 \%$ & $0.0114 \%$ & $0.0021 \%$ & $0.0052 \%$ & $0.0014 \%$ \\
\hline \multirow{6}{*}{ TPDMGM $(1,3,1)$} & \multirow{3}{*}{$\hat{x}_{i}^{(0)}(k)$} & 6284627 & 7741646 & 8656513 & 10122127 & 11653516 & 13276930 \\
\hline & & 31661 & 34487 & 37182 & 40190 & 44854 & 49926 \\
\hline & & 10602 & 10660 & 10723 & 10788 & 10853 & 10906 \\
\hline & \multirow{3}{*}{$\Delta_{s}(k)$} & $0.0388 \%$ & $0.0269 \%$ & $0.1279 \%$ & $0.2181 \%$ & $0.1254 \%$ & $0.0252 \%$ \\
\hline & & $0.0916 \%$ & $0.2530 \%$ & $0.2649 \%$ & $0.1476 \%$ & $0.0650 \%$ & $0.0190 \%$ \\
\hline & & $0.0051 \%$ & $0.0147 \%$ & $0.0126 \%$ & $0.0003 \%$ & $0.0037 \%$ & $0.0011 \%$ \\
\hline \multirow{6}{*}{ Verhulst } & \multirow{3}{*}{$\hat{x}_{i}^{(0)}(k)$} & 6300021 & 7439277 & 8713163 & 10113290 & 11623366 & 13219195 \\
\hline & & 31255 & 33880 & 36911 & 40452 & 44642 & 49673 \\
\hline & & 10604 & 10665 & 10725 & 10786 & 10846 & 10906 \\
\hline & \multirow{3}{*}{$\Delta_{s}(k)$} & $0.2838 \%$ & $3.9316 \%$ & $0.5257 \%$ & $0.1306 \%$ & $0.3838 \%$ & $0.4098 \%$ \\
\hline & & $1.1924 \%$ & $2.0104 \%$ & $0.4658 \%$ & $0.5044 \%$ & $0.4092 \%$ & $0.5255 \%$ \\
\hline & & $0.0284 \%$ & $0.0264 \%$ & $0.0320 \%$ & $0.0201 \%$ & $0.0643 \%$ & $0.0000 \%$ \\
\hline
\end{tabular}

TABLE 8: Comparisons of the prediction results of four models.

\begin{tabular}{|c|c|c|}
\hline & & 8 \\
\hline \multirow{6}{*}{$\operatorname{MGM}(1,3)$} & \multirow{3}{*}{$\widehat{x}_{i}^{(0)}(k)$} & 15995500 \\
\hline & & 55313 \\
\hline & & 10905 \\
\hline & \multirow{3}{*}{$\Delta_{p}(k)$} & $8.0721 \%$ \\
\hline & & $1.9422 \%$ \\
\hline & & $0.4291 \%$ \\
\hline \multirow{6}{*}{ DMGM $(1,3)$} & \multirow{3}{*}{$\hat{x}_{i}^{(0)}(k)$} & 15355617 \\
\hline & & 54567 \\
\hline & & 10950 \\
\hline & \multirow{3}{*}{$\Delta_{p}(k)$} & $3.7488 \%$ \\
\hline & & $0.5680 \%$ \\
\hline & & $0.0178 \%$ \\
\hline
\end{tabular}


TABLE 8: Continued.

\begin{tabular}{|c|c|c|}
\hline & & 8 \\
\hline \multirow{6}{*}{ TPDMGM $(1,3,1)$} & \multirow{3}{*}{$\widehat{x}_{i}^{(0)}(k)$} & 14702144 \\
\hline & & 55060 \\
\hline & & 10952 \\
\hline & \multirow{3}{*}{$\Delta_{p}(k)$} & $0.6663 \%$ \\
\hline & & $1.4752 \%$ \\
\hline & & $0.0013 \%$ \\
\hline \multirow{6}{*}{ Verhulst } & \multirow{3}{*}{$\widehat{x}_{i}^{(0)}(k)$} & 16539411 \\
\hline & & 63531 \\
\hline & & 11025 \\
\hline & \multirow{3}{*}{$\Delta_{p}(k)$} & $11.7470 \%$ \\
\hline & & $17.0881 \%$ \\
\hline & & $0.6691 \%$ \\
\hline
\end{tabular}

TABLE 9: Comparisons of the average simulation/prediction errors of four models.

\begin{tabular}{|c|c|c|c|c|c|}
\hline Model & $x_{i}$ & $\bar{\Delta}_{s}(\%)$ & $\bar{\Delta}_{A s}(\%)$ & $\bar{\Delta}_{p}(\%)$ & $\bar{\Delta}_{A p}(\%)$ \\
\hline \multirow{3}{*}{$\operatorname{MGM}(1,3)$} & $x_{1}$ & 1.3499 & \multirow{3}{*}{0.8768} & 8.0721 & \multirow{3}{*}{3.4811} \\
\hline & $x_{2}$ & 1.0821 & & 1.9422 & \\
\hline & $x_{3}$ & 0.1983 & & 0.4291 & \\
\hline \multirow{3}{*}{ DMGM $(1,3)$} & $x_{1}$ & 0.6941 & \multirow{3}{*}{0.3983} & 3.7488 & \multirow{3}{*}{1.4449} \\
\hline & $x_{2}$ & 0.4940 & & 0.5680 & \\
\hline & $x_{3}$ & 0.0068 & & 0.0178 & \\
\hline \multirow{3}{*}{ TPDMGM $(1,3,1)$} & $x_{1}$ & 0.0937 & \multirow{3}{*}{0.0801} & 0.6663 & \multirow{3}{*}{0.7143} \\
\hline & $x_{2}$ & 0.1402 & & 1.4752 & \\
\hline & $x_{3}$ & 0.0063 & & 0.0013 & \\
\hline \multirow{3}{*}{ Verhulst } & $x_{1}$ & 0.9442 & \multirow{3}{*}{0.6080} & 11.7470 & \multirow{3}{*}{9.8347} \\
\hline & $x_{2}$ & 0.8512 & & 17.0881 & \\
\hline & $x_{3}$ & 0.0285 & & 0.6691 & \\
\hline
\end{tabular}

TABLE 10: Comparison of modeling results among four prediction models.

\begin{tabular}{|c|c|c|c|c|c|}
\hline \multirow{2}{*}{ Case } & \multirow{2}{*}{ Index } & \multicolumn{4}{|c|}{ Model } \\
\hline & & $\operatorname{MGM}(1,3)(\%)$ & DMGM $(1,3)(\%)$ & TPDMGM $(1,3,1)(\%)$ & Verhulst (\%) \\
\hline \multirow{2}{*}{ Case 1} & $\bar{\Delta}_{A s}$ & 0.8108 & 0.0644 & 0.0594 & 3.4344 \\
\hline & $\bar{\Delta}_{A p}^{A s}$ & 1.8119 & 0.9428 & 0.9029 & 3.9838 \\
\hline \multirow{2}{*}{ Case 2} & $\bar{\Delta}_{A s}$ & 0.8768 & 0.3983 & 0.0801 & 0.6080 \\
\hline & $\bar{\Delta}_{A p}^{A s}$ & 3.4811 & 1.4449 & 0.7143 & 9.8347 \\
\hline
\end{tabular}

$(1, m, \varphi)$ model. From the aspect of the theoretical model and case study, the simulation and prediction accuracy in the TPDMGM $(1, m, \varphi)$ model are the best among four models. More important, the TPDMGM $(1, m, \varphi)$ model is compatible with the MGM $(1, m)$ model and the $\operatorname{DMGM}(1, m)$ model, which indicates the structure of the TPDMGM $(1, m, \varphi)$ model is reasonable and effective.

Therefore, after comparing the performance among these four models, the TPDMGM $(1, m, \varphi)$ model may be an optimal option to predict the system variables with mutual relation, mutual restriction, and time development trend characteristic.

\section{Conclusions}

This paper proposes a novel adaptive and high precision grey prediction model named DMGM $(1, m)$ model and its optimized model TPDMGM $(1, m, \varphi)$ model for dealing with the prediction problem of the multivariable system. The novel model can overcome the defects existing in the traditional MGM $(1, m)$ model in two aspects: first, utilizing the transformation relationship between background value and first-order accumulated sequence to obtain the definition equation of the DMGM $(1, m)$ model, so as to eliminate the jumping error in the $\operatorname{MGM}(1, m)$ model; next, by introducing a time polynomial term into the $\operatorname{DMGM}(1, m)$ model to describe the time development trend characteristics of system variables, the optimized DMGM $(1, m)$ model, abbreviated as TPDMGM $(1, m, \varphi)$ model, is obtained. As a result, both the DMGM $(1, m)$ model and the TPDMGM $(1, m, \varphi)$ model can be applied to predict the system variables with interrelation and mutual restriction characteristics, while the TPDMGM $(1, m, \varphi)$ model has a higher modeling accuracy in theoretical due to its perfect 
structure. The simulations for the illustrative case studies about deformation of foundation pit prediction and Henan's vehicle ownership prediction have been carried out to evaluate the proposed grey prediction model in comparison with the traditional grey model. The results verify that the TPDMGM $(1, m, \varphi)$ model can not only overcome the defects in the MGM $(1, m)$ model but also reflect the superiority for predicting the variables which have the mutual relation, mutual restriction, and time development trend feature.

However, the current study of the TPDMGM $(1, m, \varphi)$ model is not comprehensive. Problem, such as the intelligent algorithm optimization of parameter $\varphi$, has not been discussed in this paper. Therefore, how to construct a constraint equation and use intelligent optimization algorithms to solve the optimal parameters $\varphi$ is the next step of our work.

\section{Data Availability}

The data used to support the findings of this study are included within the article.

\section{Conflicts of Interest}

The authors declare that there are no conflicts of interest regarding the publication of this paper.

\section{Acknowledgments}

This research was supported by the Soft Science Research Plan Project of Henan Province (No. 182400410375); Humanities and Social Sciences Research General Project of Henan Colleges and Universities (No. 2020-ZDJH-140); and Key Scientific Research Projects of Colleges and Universities in Henan Province (No. 20A630015).

\section{References}

[1] J. L. Deng, “The control problems of grey systems," Systems \& Control Letters, vol. 1, no. 5, pp. 288-294, 1982.

[2] W. J. Zhou, H. R. Zhang, Y. G. Dang, and Z. X. Wang, "New information priority accumulated grey discrete model and its application," Chinese Journal of Management Science, vol. 25, no. 8, pp. 140-148, 2017.

[3] B. Zeng, S. F. Liu, Y. Bai, and M. Zhou, "Grey system modeling technology for early prediction and warning of human diseases," Chinese Journal of Management Science, vol. 28, no. 1, pp. 144-152, 2020.

[4] B. Zeng, H. Li, and X. Ma, "A novel multi-variable grey forecasting model and its application in forecasting the grain production in China," Computers \& Industrial Engineering, vol. 150, Article ID 106915, 2020.

[5] P.-P. Xiong, S. Huang, M. Peng, and X.-h. Wu, "Examination and prediction of fog and haze pollution using a multi-variable grey model based on interval number sequences," $A p$ plied Mathematical Modelling, vol. 77, pp. 1531-1544, 2020.

[6] Z. S. Meng, X. T. Liu, K. D. Yin, X. M. Li, and X. C. Guo, "Forecasting China's energy intensity by using an improved DVCGM $(1, N)$ model considering the hysteresis effect," Grey Systems: Theory and Application, vol. 11, 2020.

[7] L. Xi, S. Ding, N. Xu, and P. P. Xiong, "Research on optimization of non-equidistant GM $(1,1)$ model based on the principle of new information priority," Control and Decision, vol. 34, no. 10, pp. 2221-2228, 2019.

[8] Y. Li and J. Li, "Study on unbiased interval grey number prediction model with new information priority," Grey Systems: Theory and Application, vol. 10, no. 1, pp. 1-11, 2020.

[9] G. Y. Zou and Y. Wei, "Generalized discrete grey model and its application," Systems Engineering-Theory \& Practice, vol. 40, no. 3, pp. 736-747, 2020.

[10] J. Zhai, J. M. Sheng, and Y. J. Feng, "The Grey Model MGM (1, $N)$ and its application," Systems Engineering-Theory \& Practice, vol. 5, pp. 110-114, 1997.

[11] J. Dai, H. J. Liu, Y. N. Sun, and M. Wang, "An optimization method of multi-variable MGM $(1, m)$ Prediction model's background value"' Journal of Grey System, vol. 30, no. 1, pp. 221-238, 2018.

[12] L. Wu, X. Gao, Y. Xiao, Y. Yang, and X. Chen, "Using a novel multi-variable grey model to forecast the electricity consumption of Shandong Province in China," Energy, vol. 157, pp. 327-335, 2018.

[13] H. X. Wang and L. D. Zhao, "A nonhomogeneous multivariable grey prediction NMGM modeling mechanism and its application," Mathematical Problems in Engineering, vol. 2018, Article ID 687492, 8 pages, 2018.

[14] P. P. Xiong, X. Zou, and Y. Yang, "The nonlinear time lag multivariable grey prediction model based on interval grey numbers and its application," Natural Hazards, vol. 107, 2021.

[15] P. P. Xiong, W. Y. Yuan, L. L. Ye, and J. X. Zou, "Construction of gray MGM $(1, m, N)$ model and its prediction application on haze," Systems Engineering-Theory \& Practice, vol. 40, no. 3, pp. 771-782, 2020.

[16] P. N. Tan, M. Steinbach, and V. Kumar, Introduction to Data Mining, pp. 106-114, Pearson Addison-Wesley, Boston, MA, USA, 2006.

[17] Z. Feng, Z. P. Li, and Y. Li, "Application of a multi-point grey model to deformation prediction of supporting structure for deep pit," Chinese Journal of Rock Mechanics and Engineering, vol. 26, no. S2, pp. 4319-4324, 2007.

[18] Henan Province Bureau of Statistics, Henan Statistical Yearbook, 2020 pages, China Statistics Press, Beijing, China, 2013. 